\title{
COMPETIÇÃO ENTRE RECUPERAÇÃO E RECRISTALIZAÇÃO DINÂMICA DO AÇO INOXIDÁVEL AUSTENIITICO ASTM F I38 UTILIZADO EM IMPLANTES ORTOPÉDICOS
}

Fabio Henrique Casarini Geronimo ' Oscar Balancin ${ }^{2}$

\section{Resumo}

O aço inoxidável austenítico ASTM FI38 é utilizado na fabricação de próteses ortopédicas por forjamento. Neste trabalho determinam-se as curvas de escoamento plástico deste aço por meio de ensaios de torção a quente em ampla faixa de temperaturas e diferentes taxas de deformação. A observação microestrutural em diferentes condições de deformação, em conjunto com a utilização da técnica EBSD (Electron Backscatter Difraction), permite calcular a fração recristalizada e identificar as proporções de contornos de alto e baixo ângulos. Tendo esse aço um nível intermediário de Energia de Falha de Empilhamento (EFE), durante o amaciamento dinâmico ocorre a competição entre os mecanismos de recuperação e de recristalização dinâmicas. Assim, este trabalho tem o intuito de identificar os possíveis mecanismos de amaciamento deste aço, bem como aponta em quais condições se tornam mais atuantes.

Palavras-chave: Torção a quente; Recristalização dinâmica; Aço austenítico, EBSD.

\section{COMPETITION BEETWEN DYNAMIC RECUPERATION AND RECRYSTALLIZATION OF ASTM F I38 AUSTENITIC STAINLESS STEEL UTILIZED IN MEDICAL DEVICES}

\begin{abstract}
\section{INTRODUÇÃO}

Durante a manufatura de implantes ortopédicos metálicos, os materiais passam por um processo de forjamento a quente. Neste caso, três fenômenos controlam o comportamento mecânico durante a deformação: encruamento, recuperação dinâmica e recristalização dinâmica. Inicialmente ocorre $\circ$ encruamento e, em seguida, de acordo com as características de cada material o amaciamento é promovido via recuperação e recristalização. Estes mecanismos de amaciamento agem no sentido de dissipar a energia armazenada durante $\circ$ encruamento através do rearranjo e aniquilação das discordâncias. ${ }^{(1,2)}$
\end{abstract}

ASTM F I 38 austenitic stainless steel has being used in the manufacture of orthopedical devices by hot forging. In this work, the flow stress curves are determined by hot torsion tests in a wide range of temperatures and strain rates. With the observed microestrutural evolution by optical microscopy in different hot forming conditions in addiction with EBSD (Electron Backscatter Diffraction) techniques it is possible to obtained the recrystallized volume fraction and the misorientation angles of the samples. Due to the intermediate level of stacking fault energy of this material, during the dynamic softening occurs a competition between recrystallization and recovery. The aim of this work is to identify the softening mechanisms in this stainless steel, as well as in which hot work conditions they become more active.

Key words: Hot torsion; Dynamic recrystallization; Austenitic stainless steel; EBSD.

A recuperação e a recristalização podem ocorrer em conjunto durante $O$ amaciamento dinâmico de um metal deformado. A proporção entre a ocorrência destes mecanismos depende das características do material (especialmente a energia de falha de empilhamento) e das condições de conformação, como taxa de deformação e temperatura. ${ }^{(3,4)} A$ energia de falha de empilhamento de um material depende de sua composição química. Seu cálculo pode ser feito por meio da técnica de difração de raios $X$ e causa influência direta no escorregamento cruzado e na escalagem de discordâncias. ${ }^{(5)}$

\footnotetext{
'Engenheiro de Materiais, Doutorando em Ciências e Engenharia de Materiais, Universidade Federal de São Carlos - UFSCar, Rod. Washington Luís, km 235, SP-310, CEP 13565-905, São Carlos, SP, Brasil. E-mail: fabiogeronimo@bol.com.br

${ }^{2} \mathrm{PhD}$ em Metalurgia, Professor Doutor do Departamento de Engenharia de Materiais, Universidade Federal de São Carlos - UFSCar, Rod. Washington Luís, km 235, SP-310, CEP 13565-905, São Carlos, SP, Brasil.E-mail: balancin@ufscar.br
} 
Em metais com valores elevados de energia de falha de empilhamento, as discordâncias parciais caminham mais próximas umas das outras e por isso movem-se mais facilmente através dos mecanismos de escalagem e escorregamento cruzado. ${ }^{(6)}$ Neste caso há um aumento na taxa de aniquilação das discordâncias, diminuindo, assim, a taxa de encruamento até se atingir um ponto de igualdade entre geração e aniquilação desses defeitos, iniciando um regime de estado estacionário. A partir desse ponto os grãos são deformados e os subgrãos tornam-se constantes em forma e tamanho. ${ }^{(1,7)}$

Em metais com baixas e médias energias de falha de empilhamento, as discordâncias parciais caminham mais distantes umas das outras, aumentando a dificuldade de ocorrer o rearranjo e aniquilação desses defeitos somente através da recuperação. ${ }^{(3,4)}$ Com isso, a taxa de aniquilação é menor que a taxa de geração de discordâncias, aumentando a densidade de discordâncias nas subestruturas formadas pela recuperação e, consequentemente, a quantidade de energia armazenada no material, até que se atinge um valor crítico onde se ativam os mecanismos de nucleação da recristalização dinâmica. ${ }^{(8,9)}$

Como o aço inoxidável austenitico ASTM F 138 apresenta um valor intermediário de energia de falha de empilhamento, em torno de $78 \mathrm{~mJ} / \mathrm{m}^{2},{ }^{(5)}$ a competição entre os mecanismos de amaciamento como recuperação e recristalização é favorecida durante o seu processamento a quente. ${ }^{(3,10)}$

Neste trabalho, investigam-se por meio de ensaios de torção a quente, o comportamento mecânico e a evolução microestrutural deste material. As caracterizações microestrutural e mecânica dos processos de amaciamento dinâmicos são de extrema importância, pois o conhecimento e identificação destes mecanismos permitirão um melhoramento considerável na qualidade e no custo da produção de próteses com o aço F I 38 .

\section{MATERIAIS E MÉTODOS}

O material utilizado nesta pesquisa é um aço inoxidável com estrutura austenítica em todas as condições de trabalho termomecânico aplicadas neste estudo. É derivado do aço inoxidável 316 e produzido no Brasil pela Villares Metals S. A. pelo método de refusão por escória eletrocondutora. É denominado internacionalmente como ASTM F I 38 e, pelas normas brasileiras, como ABNT NBR ISO 5832-I. A composição química do material fornecida pelo fabricante está apresentada na Tabela I. Os corpos de prova foram usinados com diâmetro útil de $8 \mathrm{~mm}$ e comprimento útil de $12 \mathrm{~mm}$.
Neste trabalho, a deformação do material foi feita numa máquina de torção a quente projetada e construída no Laboratório de Processamentos Termomecânicos (TermoMec) da UFSCar. Foram feitos ensaios de torção a quente isotérmicos contínuos em temperaturas entre $900^{\circ} \mathrm{C}$ e $1.200^{\circ} \mathrm{C}$ e taxas de deformação entre $0,01 \mathrm{~s}^{-1}$ e $10 \mathrm{~s}^{-1}$. O corpo de prova foi aquecido com uma taxa de $2^{\circ} \mathrm{C} / \mathrm{s}$ até a temperatura de $1.200^{\circ} \mathrm{C}$ e mantido nesta temperatura por $300 \mathrm{~s}$ para completa homogeneização (resultando num tamanho médio de grão $\left.D_{\text {inicial }}=85,1 \mu \mathrm{m}\right)$. Em seguida, foi resfriado também com uma taxa de $2^{\circ} \mathrm{C} / \mathrm{s}$ até a temperatura de ensaio. Ao final da deformação, foi realizado um resfriamento rápido a fim de congelar a microestrutura para análise metalográfica.

Em algumas condições de processamento, para se conhecer a evolução microestrutural deste material, foram feitos também ensaios isotérmicos interrompidos em pontos estratégicos (Figura I), como a deformação crítica para início da recristalização dinâmica $\left(\varepsilon_{c}\right)$, a deformação de pico $\left(\varepsilon_{\mathrm{p}}\right)$, alguns pontos no estado estacionário da deformação $\left(\varepsilon_{\mathrm{ss}}\right)$ e na deformação final $\left(\varepsilon_{\mathrm{f}}\right)$. Em seguida, as microestruturas resultantes foram analisadas por microscopia óptica, e posteriormente, por microscopia eletrônica de varredura via EBSD, para identificar os mecanismos de amaciamento atuantes e sua fração recristalizada dinamicamente. ${ }^{(3,4,11)}$ Para a análise metalográfica, as amostras foram lixadas, polidas com alumina $(\mathrm{I} \mu \mathrm{m}) \mathrm{e}$ atacadas eletroquimicamente com ácido nítrico (P.A.) por alguns segundos. Para a realização dos EBSDs, as amostras receberam um polimento extra com sílica coloidal em uma politriz vibratória por algumas horas. As medidas de tamanho de grão foram feitas pelo método computacional com a utilização do software AnalySis ${ }^{\circledR}$ utilizando-se um microscópio óptico acoplado a uma câmera de vídeo, ligados a um computador.

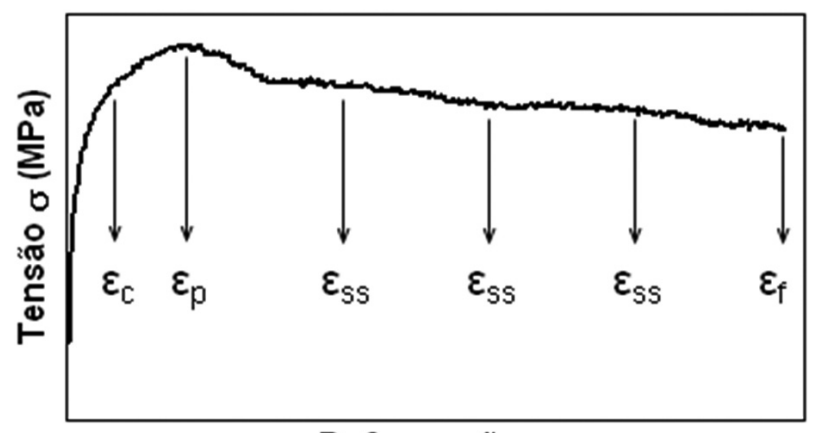

Deformação $\varepsilon$

Figura I. Curva ilustrando os pontos específicos para os ensaios interrompidos.

Tabela I. Composição química do aço inoxidável F I 38 (\% em massa)

\begin{tabular}{cccccccccc}
\hline $\mathbf{C}$ & $\mathbf{S i}$ & $\mathbf{M n}$ & $\mathbf{P}$ & $\mathbf{S}$ & $\mathbf{C r}$ & $\mathbf{M o}$ & $\mathbf{N i}$ & $\mathbf{C u}$ & $\mathbf{N}$ \\
\hline 0,011 & 0,19 & 1,75 & 0,019 & $<0,001$ & 17,30 & 2,77 & 14,30 & 0,09 & 0,079 \\
\hline
\end{tabular}




\section{RESULTADOS}

\section{I Curvas de Escoamento Plástico}

Os gráficos de tensão versus deformação equivalentes $(\sigma \times \varepsilon)$ obtidos nos ensaios estão separados em quatro grupos, de acordo com as taxas de deformação utilizadas $\left(0.01 \mathrm{~s} \mathrm{~s}^{-1}, 0.1 \mathrm{~s}^{-1}, \mathrm{I} \mathrm{s}^{-1}\right.$ e $\left.10 \mathrm{~s}^{-1}\right)$, e apresentados na Figura $2(\mathrm{a}-\mathrm{d})$. Cada grupo contém as curvas dos ensaios para as temperaturas de $900^{\circ} \mathrm{C}, 950^{\circ} \mathrm{C}, 1.000^{\circ} \mathrm{C}, 1.050^{\circ} \mathrm{C}$, I. $100^{\circ} \mathrm{C}$ e $1.200^{\circ} \mathrm{C}$ aplicadas neste trabalho, totalizando 24 ensaios. As curvas de escoamento plástico podem fornecer informações importantes quanto ao mecanismo de amaciamento atuante, de acordo com sua forma.

Curvas que apresentam um pico de tensão anterior ao estado estacionário indicam a ocorrência da recristalização dinâmica. ${ }^{(8,9)}$ Curvas onde não há um pico evidente anterior ao estado estacionário indicam que o mecanismo predominante é a recuperação. Neste caso, a curva é conhecida como flat-top, ${ }^{(3)}$ onde após o encruamento inicial atinge-se o estado estacionário. Em seguida, há apenas uma pequena mudança na tensão com $o$ aumento da deformação. Este comportamento da curva é um forte indício que o único mecanismo de amaciamento ocorrente é a recuperação. ${ }^{(12)}$

Observa-se (Figura 2) que a tensão aumenta com o aumento da deformação até atingir um valor de pico. Em seguida, nas condições de maior temperatura e menores taxas de deformação, o valor da tensão diminui alcançando o estado estacionário até o término dos ensaios, sendo este comportamento típico de metais que apresentam o processo de amaciamento através da recristalização dinâmica. ${ }^{(8,9,13,14)}$ Em alguns ensaios com temperaturas baixas e intermediárias, as curvas apresentam um formato do tipo flat-top, onde, após o pico, a queda de tensão é pequena e - estado estacionário é alcançado com níveis próximos da tensão máxima. Nestes casos os mecanismos de amaciamento dinâmicos ocorrem em conjunto e a recristalização não se completa.

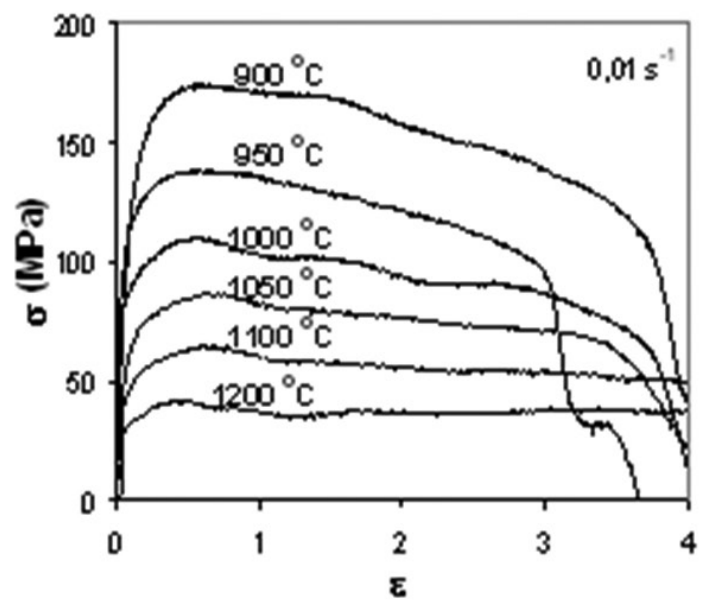

(a)

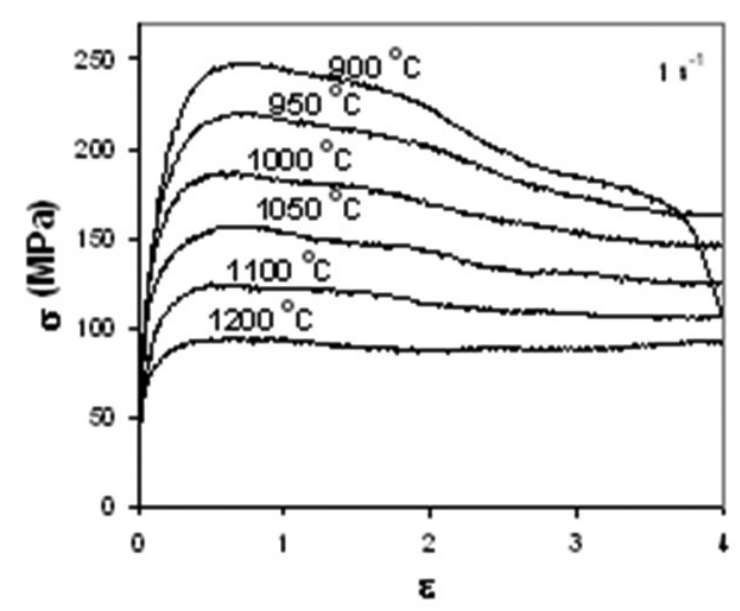

(c)

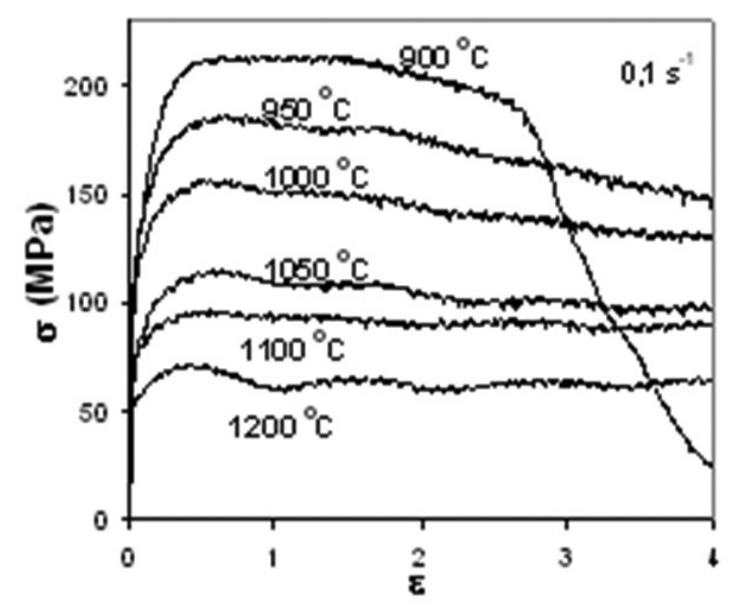

(b)

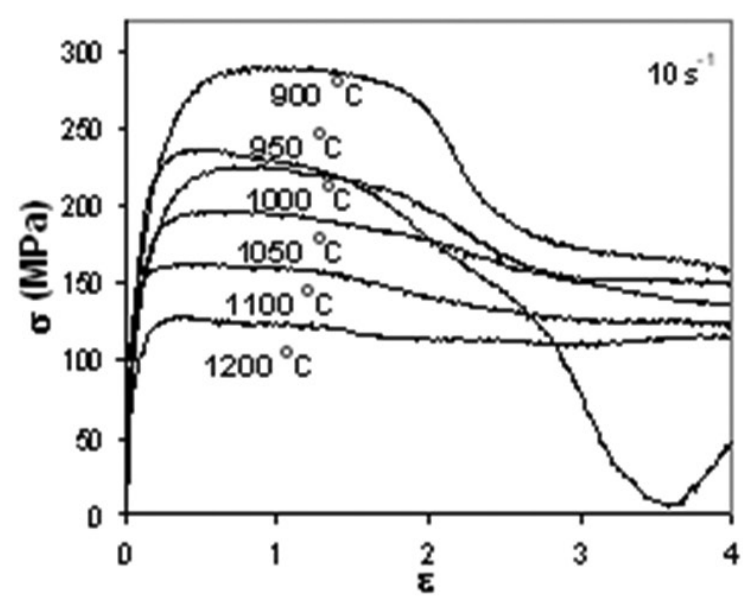

(d)

Figura 2. Curvas de escoamento plástico do aço F I38. 
Entretanto, estudos anteriores mostram que a ausência do pico na curva de escoamento plástico não indica necessariamente a ausência da recristalização dinâmica. ${ }^{(15)}$ Neste caso, deve-se identificar o ponto de inflexão nas curvas de taxa de encruamento $\theta$ versus tensão equivalente $\sigma$ (Figura 3), indicando que a recristalização ocorre, mesmo que em pequena proporção. ${ }^{(3)}$ Este método analítico, desenvolvido por Poliak e Jonas ${ }^{(15)}$, é muito útil nestes casos e também é utilizado para identificar os valores das tensões críticas, de pico e de estado estacionário.

\subsection{Microestruturas}

Uma análise visual das amostras por microscopia óptica permitiu a constatação do amaciamento dinâmico em todas as condições de ensaios, como pode ser visto na Figura 4. O tamanho de grão austenítico inicial foi reduzido de cerca de 85 , I $\mu \mathrm{m}$ para valores entre aproximadamente $2 \mu \mathrm{m}$ e $18 \mu \mathrm{m}$ (Tabela 2). Entretanto, juntamente com os valores médios dos grãos resultantes, é possível identificar dois grupos distintos nesta tabela, conforme o mecanismo preferencial de amaciamento atuante.

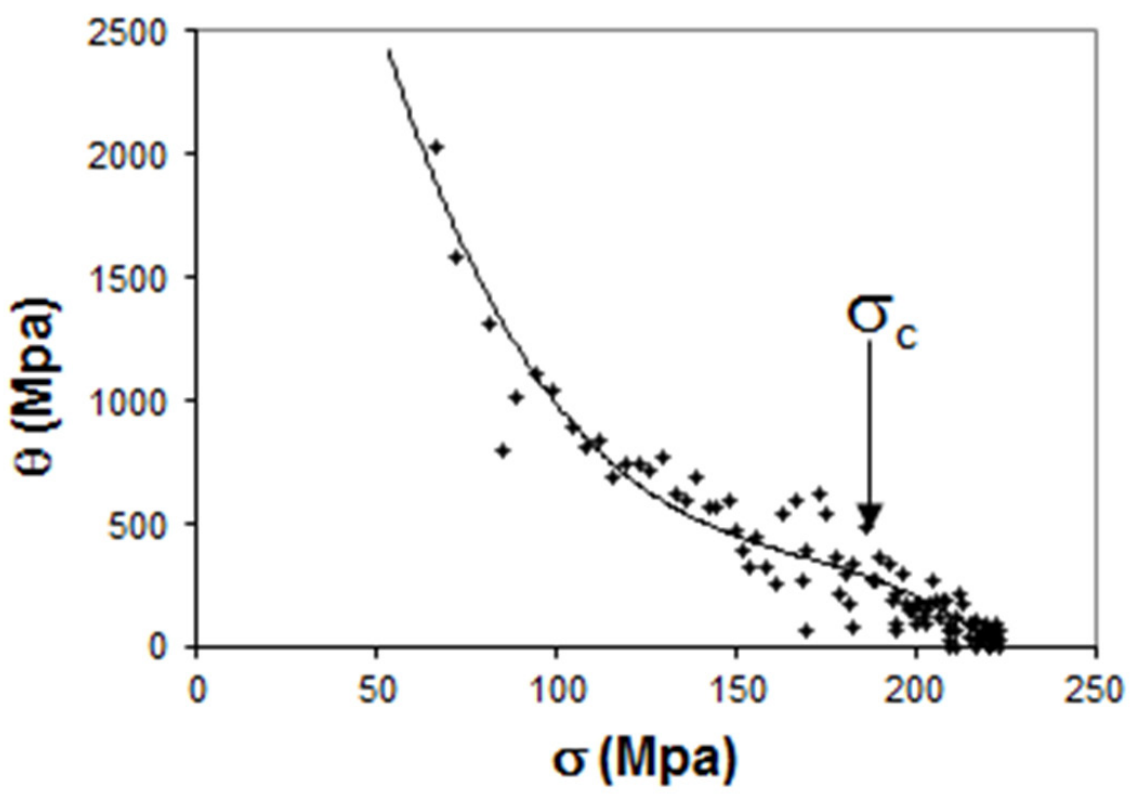

Figura 3. Curva de $\theta$ versus $\sigma$ em $\mathrm{T}=1.000^{\circ} \mathrm{C}$ e $\dot{\varepsilon}=10 \mathrm{~s}^{-1}$, indicando o ponto de inflexão característico da tensão crítica para início da recristalização dinâmica.
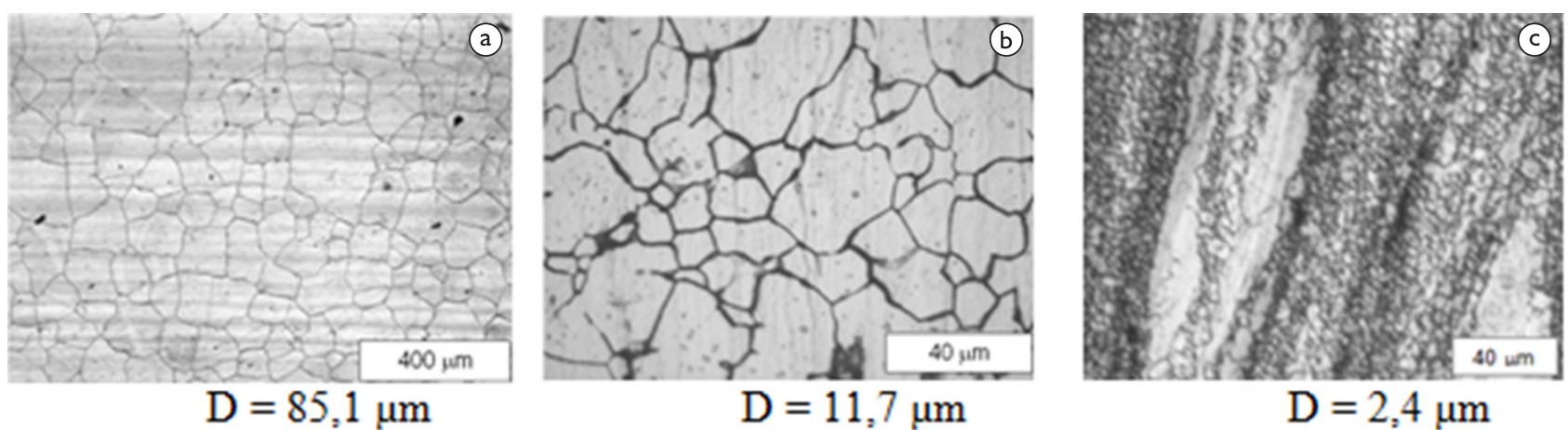

Figura 4. Microestruturas das amostras observadas nas condições de: (a) inicial, (b) $\mathrm{I} .200^{\circ} \mathrm{C}$ e I s${ }^{-1}$ e (c) $900^{\circ} \mathrm{C}$ e 0,0 l s ${ }^{-1}$ e respectivos tamanhos médios de grãos.

Tabela 2. Tamanhos de grão medidos em todas as condições de ensaio

\begin{tabular}{|c|c|c|c|c|c|c|}
\hline \multirow{2}{*}{$\begin{array}{c}\dot{\varepsilon} \\
\left(s^{-1}\right)\end{array}$} & \multicolumn{6}{|c|}{$\mathbf{T}\left({ }^{\circ} \mathbf{C}\right)$} \\
\hline & 900 & 950 & 1.000 & 1.050 & 1.100 & 1.200 \\
\hline 0,01 & 2,4 & 3,1 & 6,2 & 10,0 & 12,1 & 17,7 \\
\hline 0,1 & 1,9 & 2,6 & 3,4 & 4,6 & 6,2 & 10,0 \\
\hline I & 2,5 & 2,5 & 3,3 & 4,1 & 8,3 & $1 \mathrm{I}, 7$ \\
\hline 10 & 4,1 & 4,5 & 7,3 & 7,5 & 8,0 & 11,0 \\
\hline
\end{tabular}


O grupo em destaque representa os ensaios onde as condições são favoráveis à recristalização dinâmica (altas temperaturas e baixas taxas de deformação). Nestes casos, o amaciamento é eficiente e a microestrutura apresenta-se completamente recristalizada (Figura 4b). Todos os grãos austeníticos resultantes têm morfologia equiaxial, porém com grande variação em seu tamanho.

No outro grupo, devido às condições de deformação (baixas temperaturas e altas taxas de deformação), a recuperação e a recristalização dinâmicas atuam em conjunto e o amaciamento éparcial (Figura 4c). Neste caso, a recristalização ocorre preferencialmente através do mecanismo conhecido como necklace, onde a formação de novos grãos austeníticos ocorre na vizinhança dos contornos de grãos deformados e em contornos de maclas, decorrentes de flutuações na sua forma.

\subsection{Evolução Microestrutural}

Depois de identificar os grupos distintos de microestruturas resultantes, foram feitos ensaios isotérmicos contínuos interrompidos em pontos pré-definidos das curvas de escoamento conforme identificado na Figura I. Em todos os casos, as microestruturas foram analisadas por microscopia óptica e revelam o avanço do amaciamento durante a aplicação do esforço mecânico.

$\mathrm{Na}$ Figura 5, que representa a amostra ensaiada sob $\mathrm{T}=900^{\circ} \mathrm{C}$ e $\dot{\varepsilon}=1 \mathrm{~s}^{-1}$ em condições de recristalização parcial, percebe-se um avanço lento da recristalização. $\mathrm{Na}$ deformação crítica há pouca formação de contornos serrilhados e embojamento. No pico de tensão os primeiros colares de recristalização ainda não estão formados. Nos pontos intermediários inicia-se a formação de novos grãos, porém ainda percebe-se uma forte ação do encruamento e, como consequência, da recuperação dinâmica. Somente na condição final é que a microestrutura mostra-se homogênea, aparentemente recristalizada e com grãos austeníticos pequenos ( $D \sim 2,5 \mu \mathrm{m}$ ).

A Figura 6 representa a amostra deformada sob $\mathrm{T}=1.200^{\circ} \mathrm{C}$ e $\dot{\varepsilon}=1 \mathrm{~s}^{-1}$ pertencente ao grupo que apresenta recristalização completa. Neste caso percebe-se que os contornos de grão ensaiados até a deformação crítica apresentam serrilhamento, o que leva à formação dos colares na deformação de pico. A microestrutura já se apresenta homogênea em torno de $\varepsilon=2,0 \mathrm{e}$ permanece assim até $\circ$ final do experimento, indicando a recristalização dinâmica total do material nestas condições, com grãos austeníticos maiores e tamanho médio de $\mathrm{D} \sim \mathrm{II}, 7 \mu \mathrm{m}$.

\subsection{EBSD}

As amostras submetidas ao EBSD foram selecionadas de acordo com os resultados microestruturais citados no item 3.3. As amostras foram analisadas na condição final de deformação $(\varepsilon=4,0)$ e estão representadas nas Figuras 7 e 8.

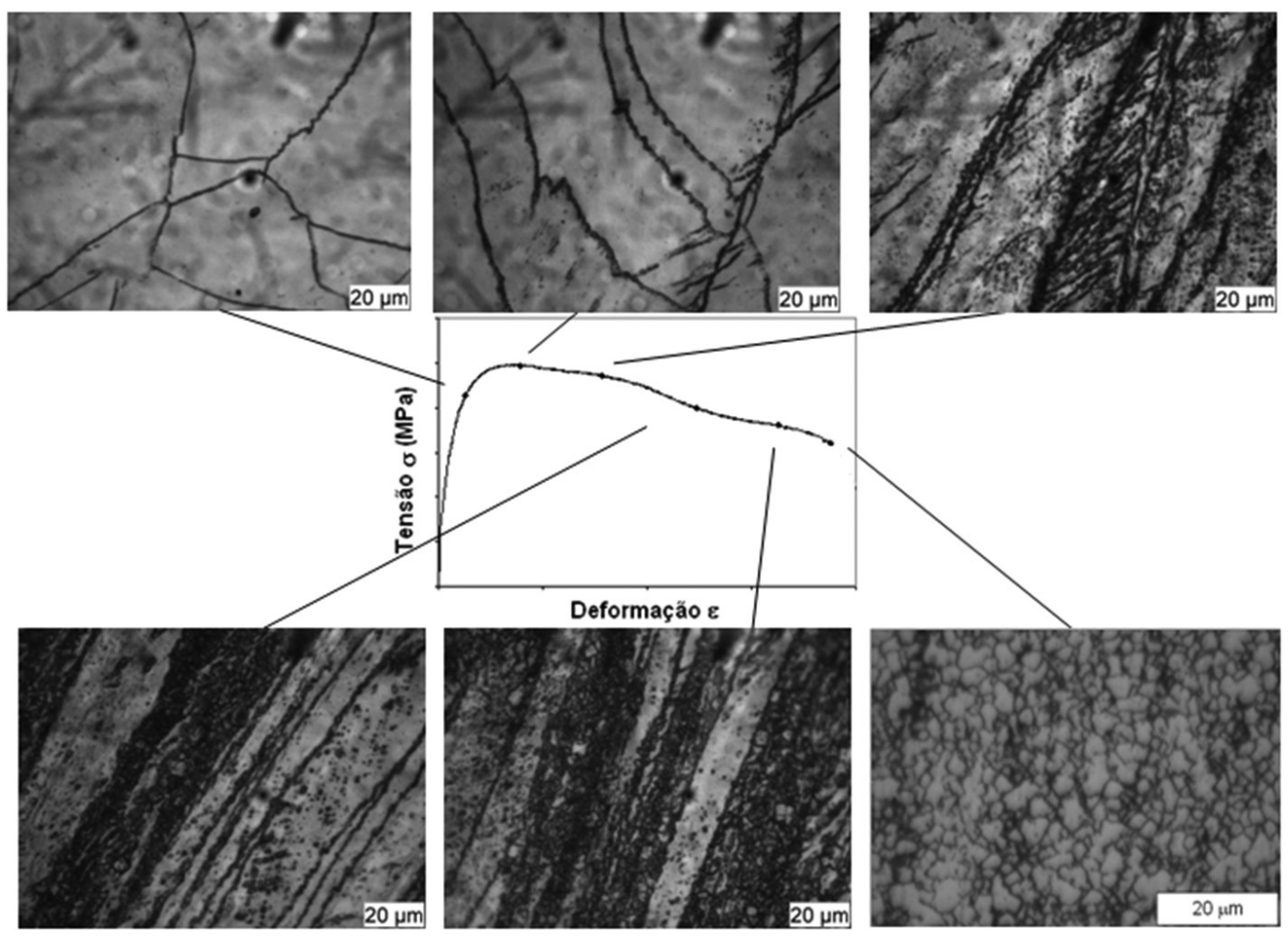

Figura 5. Evolução microestrutural da amostra para $\mathrm{T}=900^{\circ} \mathrm{C}$ e $\dot{\varepsilon}=1 \mathrm{~s}^{-1}$. 


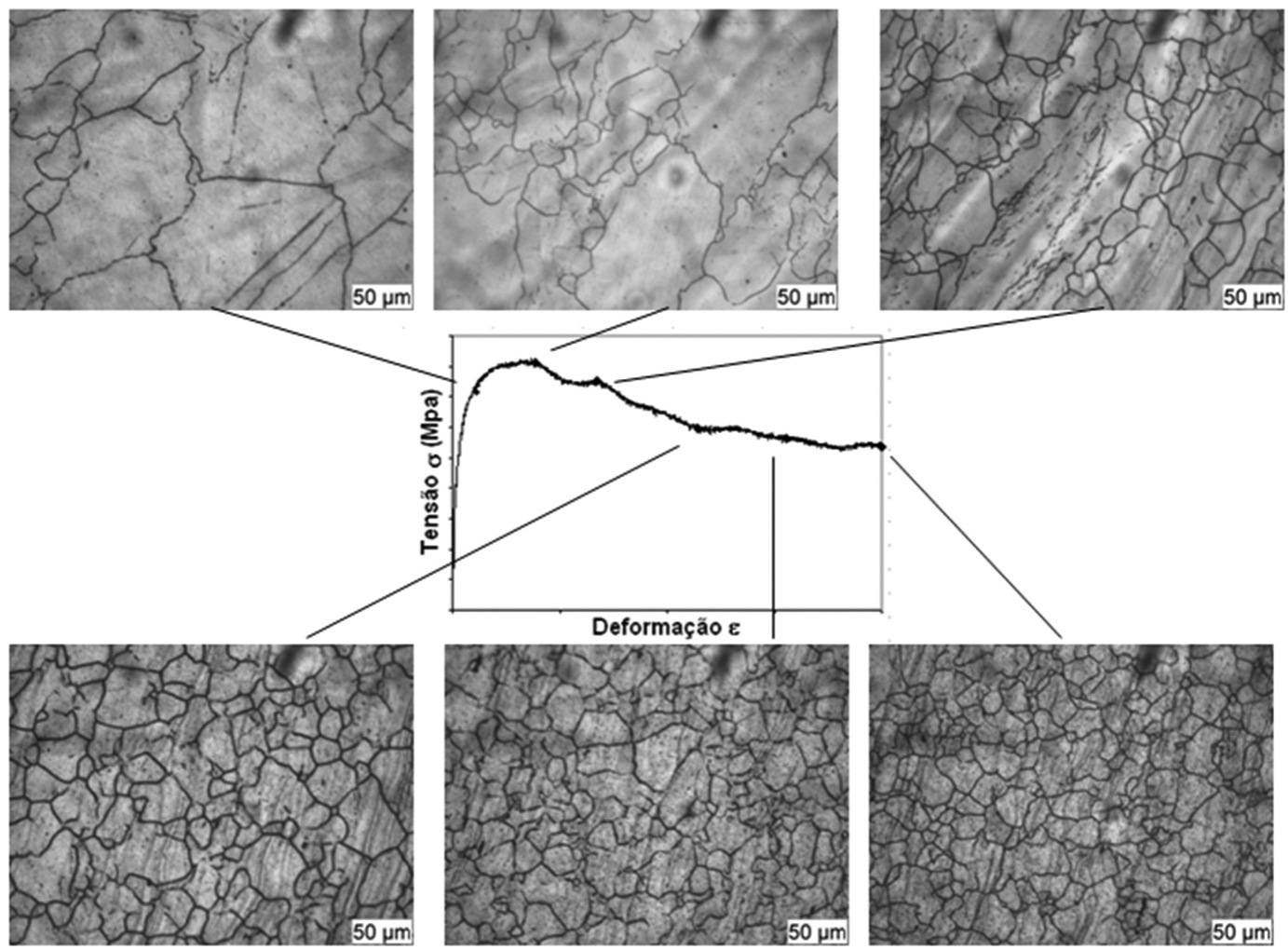

Figura 6. Evolução microestrutural da amostra para $\mathrm{T}=1.200^{\circ} \mathrm{C}$ e $\dot{\varepsilon}=1 \mathrm{~s}^{-1}$.

Nos dois casos, as amostras apresentam evidências da presença de ambos os mecanismos de amaciamento dinâmicos, ou seja, recuperação e recristalização. Os contornos de grão em preto são vizinhanças com ângulo de orientação maior que $15^{\circ}$, caracterizando a recristalização dinâmica, enquanto que os contornos em branco têm ângulo de orientação entre $2^{\circ}$ e $15^{\circ}$, caracterizando a recuperação dinâmica. ${ }^{(16)}$

$\mathrm{Na}$ Figura 7, percebe-se que a quantidade de grãos recristalizados e recuperados é praticamente igual. $O$ cálculo estatístico realizado com o software acoplado ao EBSD indica que a fração recristalizada é de aproximadamente $53 \%$. Com isso, pode-se dizer que neste material predominam ambos os mecanismos de amaciamento dinâmicos nestas condições de conformação a quente. Na Figura 8 nota-se que a quantidade de grãos recristalizados dinamicamente é significativamente maior que os grãos recuperados. O cálculo feito pelo software revela que a fração recristalizada é de aproximadamente $85 \%$. Sendo assim, pode-se dizer que, nestas condições de conformação a quente, a recristalização dinâmica praticamente se completa.

\section{DISCUSSÃO}

Como esperado, todas as curvas de escoamento plástico apresentam um encruamento inicial e, para uma mesma taxa de deformação, a tensão máxima aumenta com o decréscimo da temperatura. Após o encruamento, as curvas exibem um ponto máximo de tensão seguido de um decréscimo decorrente do amaciamento até atingir
○ estado estacionário. Em alguns casos, principalmente em temperaturas mais baixas e intermediárias, o pico de tensão não é pronunciado. Nesses casos, o estado estacionário é atingido em valores próximos à tensão de pico e as curvas adquirem um formato do tipo flat-top, como já reportado por outros autores. ${ }^{(3,12)}$

Numa análise detalhada das curvas, pode-se dizer que a recristalização dinâmica é o mecanismo de amaciamento predominante na maioria dos casos, principalmente em temperaturas mais elevadas como $1.200^{\circ} \mathrm{C}$, $1.100^{\circ} \mathrm{C}$ e $1.050^{\circ} \mathrm{C}$. Nas condições onde as curvas apresentam o comportamento flat-top, a recristalização não é o mecanismo dinâmico predominante de amaciamento e a recuperação assume um papel importante nestes casos. Alguns autores reportam que há uma faixa de transição entre as duas situações, conforme se aumenta a taxa de deformação, ${ }^{(17)}$ fato esperado também para o aço $\mathrm{F} I 38$.

Como mostrado anteriormente, ${ }^{(15)}$ a recristalização dinâmica pode ocorrer mesmo se não forem observados picos de tensão nas curvas de escoamento plástico. Nestes casos, a análise das curvas da taxa de encruamento versus tensão equivalente mostram que em todas as situações de conformação deste trabalho, foram encontrados os pontos de inflexão nas curvas, caracterizando a recristalização dinâmica, mesmo que parcialmente em alguns casos.

Entretanto, após a constatação do comportamento flat-top de algumas curvas, e sabendo que o aço F 138 apresenta um valor intermediário de energia de falha de empilhamento é confirmada a hipótese de que a recuperação dinâmica age de forma a competir com a recristalização. 


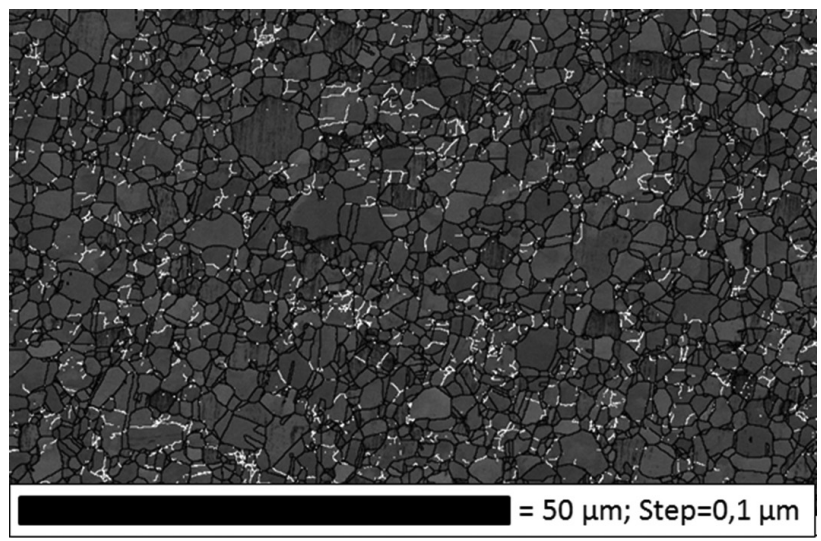

Figura 7. Representação em EBSD da amostra para $\mathrm{T}=900^{\circ} \mathrm{C}$ e $\dot{\varepsilon}=\mid \mathrm{s}^{-1}$.

Para a comprovação deste fato, foi avaliada uma amostra de cada grupo $\left(\mathrm{T}=1.200^{\circ} \mathrm{Ce} \dot{\varepsilon}=1 \mathrm{~s}^{-1} \mathrm{e} \mathrm{T}=900^{\circ} \mathrm{C}\right.$ e $\left.\dot{\varepsilon}=\mid \mathrm{s}^{-1}\right)$ quanto à sua evolução microestrutural e também analisada via EBSD. Em ambos os casos são detectados subgrãos com ângulos de orientação inferiores a $15^{\circ}$, caracterizando a recuperação dinâmica. A ocorrência de ambos os mecanismos de amaciamento dinâmicos fica mais evidenciada na condição de menor temperatura, com fração recristalizada dinamicamente de $53 \%$, enquanto que, na temperatura mais elevada, esta fração fica em torno de $85 \%$.

\section{CONCLUSÃO}

Devido ao nível intermediário da energia de falha de empilhamento, o aço F I38 apresenta seu amaciamento dinâmico através dos mecanismos de recuperação e recristalização, de acordo com as condições de trabalho

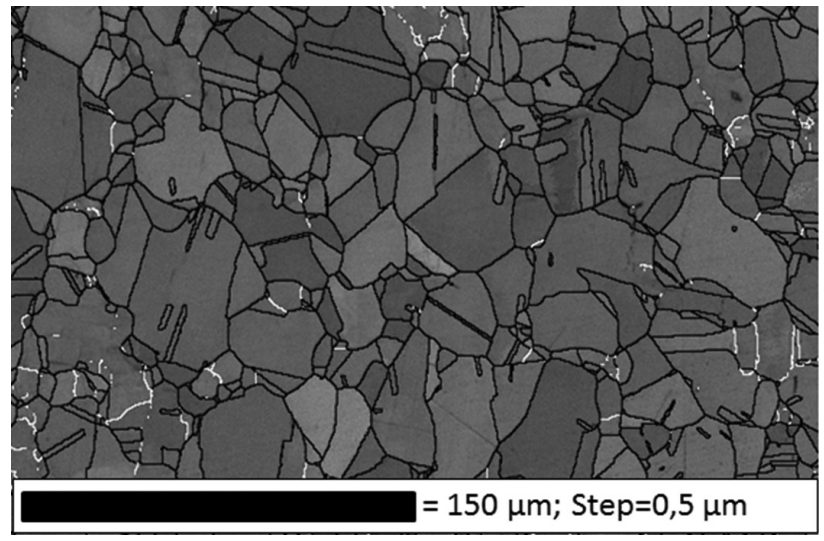

Figura 8. Representação em EBSD da amostra para $T=1200^{\circ} \mathrm{C}$ e $\dot{\varepsilon}=\mid \mathrm{s}^{-1}$.

a quente, principalmente em relação à temperatura. Em temperaturas mais elevadas o material experimenta - amaciamento preferencialmente via recristalização, enquanto que, em temperaturas mais baixas, a recuperação torna-se mais atuante. Estes fatos ficam evidenciados pelo formato característico das curvas de escoamento plástico nestas condições e comprovados através da análise da evolução microestrutural e EBSD.

\section{Agradecimentos}

Os autores agradecem à empresa Villares Metals S.A. por fornecer o material para o estudo e também ao suporte financeiro dado pela Coordenação de Aperfeiçoamento de Pessoal de Nível Superior - CAPES

\section{REFERÊNCIAS}

I McQueen HJ, Jonas JJ. Recovery and recrystallization during high temperature deformation. In: Herman H, editor. Treatise on materials science and technology. New York: Academic Press; 1975. v. 6, p. 393-493.

2 Ahlblom B, Sandstrom R. Hot workability of stainless steel: Influence of deformation parameters, microstrutural components and restoration process. Int Metals Rev. 1982; I:I-27. http://dx.doi.org/ I0. I I79/09506608279032444 I

3 Taylor AS, Hodgson PD. Dynamic behaviour of 304 stainless steel during high Z deformation. Mat Sci Eng A-Struct. 201 I;528:3310-3320. http://dx.doi.org/10.1016/j.msea.2010.12.093

4 Samantaray D. et al. Flow behavior and microstructural evolution during hot deformation of AISI type $316 \mathrm{~L}(\mathrm{~N})$ austenitic stainless steel. Mat Sci Eng A-Struct. 201 I;528:29-30. http://dx.doi.org/10.1016/j.msea.20II.08.012

5 Schramm RE, Reed RP. Stacking fault energy of seven commercial austenitic stainless steels. Metall Mater Trans A. 1975;6:1345-I35I. http://dx.doi.org/I0.1007/BF02641927

6 Dieter EG. Mechanical metallurgy. New York: Mc Graw-Hill; 1976.

7 McQueen HJ. The production and utility of recovered dislocation substructure. Metall Mater Trans A. 1977;8:807-824. http://dx.doi.org//0.1007/BF0266/562

8 Ponge D, Gottstein G. Necklace formation during dynamic recrystallization: mechanisms and impact on flow behavior. Acta Mater. 1998;46:69-80. http://dx.doi.org/10.1016/SI359-6454(97)00233-4

9 Jonas JJ, Quelennec X, Lan J, Martin E. The Avrami kinetics of dynamic recrystallization. Acta Mater. 2009;57:2748-2756. http://dx.doi.org/10.1016/j.actamat.2009.02.033 
I0 Stuwe HP, Padilha AF, Siciliano Junior F. Competition between recovery and recrystallization. Mat Sci Eng A-Struct. 2002;333:36I-7. http://dx.doi.org/I0.10I6/S092I-5093(0I)0I860-3

I I El Wahabi M, Gavard L, Cabrera JM, Prado JM, Montheillet F. EBSD study of purity effects during hot working in austenitic stainless steels. Mat Sci Eng A-Struct. 2005;393:83-90. http://dx.doi.org/10.1016/j.msea.2004.09.064

12 Davenport SB, Silk NJ, sparks CN, Sellars CM. Development of constitutive equations for modeling of hot rolling. Mater Sci Technol. 2000; I6:539-546. http://dx.doi.org/I0. I I79/026708300 I0I508045

13 Jafari M, Najafizadeh A. Correlation between Zener-Hollomon parameter and necklace DRX during hot deformation of 316 stainless steel. Mat Sci Eng A-Struct. 2009;50I:16-25. http://dx.doi.org/10.1016/j. msea.2008.09.073

14 Jafari M, Najafizadeh A, Rasti J. Dynamic recrystallization by necklace mechanism during hot deformation of 316 stainless steel. Int J Iron Steel Soc Iran. 2007;4: 16-23.

15 Poliak El, Jonas JJ. Initiation of dynamic recrystallization in constant strain rate hot deformation. ISIJ Int. 2003;43:684-69I. http://dx.doi.org/I0.2355/isijinternational.43.684

16 Doherty RD, Hughes DA, Humpheys FJ, Jonas JJ, Juul Jensen D, Kassner ME et al. Current issues in recrystallization: a review. Mat Sci Eng A-Struct. 1997;238:2 19-274. http://dx.doi.org/I0.1016/S092I-5093(97)00424-3

17 Almeida JAD, Barbosa R. Hot deformation of 304 type austenitic stainless steel at high strain rates. ISIJ Int. 2003;43:264-266. http://dx.doi.org//0.2355/isijinternational.43.264

Recebido em: 19/10/2012

Aceito em: 02/05/2013 